'Escuela de Química y Farmacia, Facultad de Química y Farmacia. Pontificia Universidad Católica de Chile. Santiago, Chile.

2Programa de Farmacología y Toxicología, Facultad de Medicina Pontificia Universidad Católica de Chile. Santiago, Chile.

${ }^{3}$ Departamento de Medicina Intensiva, Facultad de Medicina. Hospital Clínico. Pontificia

Universidad Católica de Chile. Santiago, Chile.

aPrograma de Química y

Farmacia. Tesista para optar al grado de licenciada.

bQuímico Farmacéutico. ${ }^{\circ}$ Doctora en Ciencias Farmacéuticas. ${ }^{\mathrm{d} M S C \text { FACP }}$

Trabajo no recibió financiamiento.

Los autores declaran no tener conflictos de interés.

Recibido el 27 de noviembre de 2020, aceptado el 25 de mayo de 2021.

Correspondencia a: Dr. Max Andresen $\mathrm{H}$. Departamento de Medicina Intensiva.

Facultad de Medicina.

Hospital Clínico Pontificia Universidad Católica de Chile. Marcoleta 367- Santiago de Chile andresen@med.puc.cl

\section{Determinación de factores de riesgo a reacciones adversas a medicamentos mediante farmacovigilancia intensiva en UCI}

\author{
KARIN OBREQUE ${ }^{1, \mathrm{a}}$, ROSEMARIE MELLADO ${ }^{1,2, \mathrm{~b}, \mathrm{c}}$, MAX ANDRESEN $^{3, \mathrm{~d}}$
}

\section{Risk factors for adverse drug reactions in patients admitted to intensive care units}

Background: Patients hospitalized in intensive care units (ICU) are at higher risk of having adverse drug reactions (ADR). Aim: To determine risk factors for ADR, through intensive pharmacological surveillance at the ICU. Patients and Methods: An observational, descriptive and prospective study was made, determining risk parameters in patients who experienced ADR. Results: Eighty-five patients were surveilled and 24 (28\%) had an ADR. A total of 48 drugs responsible for at least one $A D R$ were identified. Seventy-three percent $A D R$ were moderate and $27 \%$ were severe. The clinical variables significantly associated with ADR were a history of allergies, a high body mass index, the reason for admission, an APACHE II score $\geq 14$ points, the use of invasive mechanical ventilation and more than seven days of hospitalization. The pharmacological variables associated with $A D R$ were polypharmacy and medication associations and combinations. Conclusions: The identified risk factors have a great impact on pharmacokinetic and pharmacodynamic parameters, and should be considered to avoid the appearance of $A D R$.

(Rev Med Chile 2021; 149: 1258-1266)

Key words: Drug Interactions; Drug-Related Side Effects and Adverse Reactions; Polypharmacy).

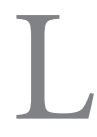

a unidad de cuidados intensivos (UCI) es un área hospitalaria destinada al manejo terapéutico de pacientes que presentan un grave compromiso a sistemas orgánicos, requiriendo de un plan terapéutico que incluye una amplia farmacoterapia ${ }^{1,2}$. El uso de medicamentos debería generar únicamente beneficios al paciente, con nulo riesgo, sin embargo, no están exentos de producir resultados negativos a la medicación (RNM), condición que puede impactar la evolución del paciente ${ }^{3-5}$.

Uno de los RNM más relevantes, se conoce como reacción adversa a medicamentos (RAM) $)^{5,6}$ y estudios fármaco-epidemiológicos han evidenciado que entre $6,1 \%$ y $18,1 \%$ de los pacientes hos- pitalizados padecería una RAM, correspondiendo $6,7 \%$ a RAM graves ${ }^{7-8}$.

Los pacientes que ingresan a UCI presentan factores de riesgo para el desarrollo de RAM, comprometiendo no solo la recuperación del paciente, sino que, además, elevando costos asociados y retrasando una eficaz y activa alta de la unidad $^{8,9}$. Existen estudios que evalúan factores de riesgo a RAM, no obstante, comprenden área pediátrica ${ }^{10,11}$ y de medicina interna ${ }^{12}$. Por este motivo, el objetivo de la presente investigación es evaluar y determinar factores de riesgo de RAM en pacientes de UCI, además, determinar el nivel de concordancia entre métodos de clasificación RAM, evaluando grado de confiabilidad entre sí. 


\section{Pacientes y Método}

\section{Modelo de estudio}

Estudio observacional, prospectivo y descriptivo, en pacientes que ingresaron a UCI del Hospital Clínico Red de Salud UC CHRISTUS, aprobado por Comité de Ética Científico de la Facultad de Medicina, acreditado por resolución $\mathrm{N}^{\circ} 018526$, durante 5 meses (agosto- enero). Se establecieron 3 etapas: primer mes, diseño de proyecto; segundo mes, corrección de falencias; $y$, finalmente, 3 meses de seguimiento a pacientes y recopilación de datos. Se incluyeron pacientes que, dentro de su farmacoterapia, recibieron medicamentos pertenecientes a: antibióticos, anticonvulsivantes, antifúngicos, sedantes y analgésicos, pues son familias farmacológicas de mayor frecuencia de administración en $\mathrm{UCI}^{13}$. Se excluyeron a pacientes cuya estadía fuera menos de $48 \mathrm{~h}$.

\section{Detección, evaluación y selección de RAM}

Se registró diariamente la evolución de pacientes mediante visitas médicas y registro de farmacoterapia administrada durante estadía en UCI. Para no omitir ninguna RAM, se establecieron señales de alerta que permitieron reconocer fácilmente una RAM, por ejemplo, suspensión abrupta de medicamentos, inclusión de fármacos adicionales al tratamiento base de patología de ingreso y cambio de farmacoterapia por otra similar, con distinto mecanismo farmacológico. Posteriormente se realizó evaluación de causalidad de la Organización Mundial de la Salud (OMS) ${ }^{14}$ y algoritmo de Naranjo ${ }^{15}$, mediante puntajes establecidos, para valorar relación causal de fármaco sospechoso y RAM. Se excluyeron las RAMs de clasificación improbable, inclasificable o dudosa, con el objetivo de no generar un sesgo positivo en el estudio.

\section{Obtención y recopilación de datos}

La obtención y recopilación de datos se realizó mediante sistema de farmacovigilancia intensiva, monitoreo sistemático de la farmacoterapia del paciente en la unidad ${ }^{16}$. Se recabó, además, información desde el equipo médico y de enfermería, exámenes de laboratorio e información de ficha médica. Los exámenes fueron aquellos solicitados por el médico tratante, no implicando otros adicionales. Se recopiló resultados mediante ficha farmacéutica digitalizada, evaluando datos antropodemográficos, datos clínicos, incluyendo, ventilación mecánica invasiva (VMI), índices de gravedad: Sequential Organ Failure Assessment (SOFA) ${ }^{16}$, Score Acute Physiology and Chronic Health Evaluation II (APACHE II) ${ }^{17}$ y datos farmacológicos (Figura 1). Se valorizó RAM según alteración fisiológica, causalidad de $\mathrm{OMS}^{14} \mathrm{y}$ algoritmo de Naranjo ${ }^{15}$, clasificación de RAM según gravedad ${ }^{10} \mathrm{y}$ mecanismo de acción del fármaco ${ }^{19}$, estableciendo nivel de concordancia entre métodos de causalidad y clasificación RAM.

\section{Estadística}

Para comprobación de normalidad y análisis estadístico se empleó el software IBM SPSS. Se empleó técnica $\mathrm{t}$ de Student $(t)$ para variables numéricas y Chi-cuadrado $\left(\chi^{2}\right)$ para variables cualitativas. Para comparar riesgo de desarrollo de RAM ante presencia de parámetros evaluados se utilizó test de riesgo relativo (RR). La potencia de asociación de parámetros, se efectuó mediante correlación de Spearman (rho) y, finalmente, para evaluar concordancia entre métodos de clasificación de RAM, se efectuó test de concordancia de Kruskall Wallis y prueba de confiabilidad $\alpha$ de Cronbach.

\section{Resultados}

\section{Generales}

Ingresaron 135 pacientes a UCI, promediando 45 pacientes/mes. Del total de ingresos, 42 pacientes se excluyeron por estadía menor a 48 h. Un pequeño porcentaje de los 42 pacientes excluidos (5 casos) no recibía medicamentos conforme a los criterios de inclusión. Finalmente, se reunieron antecedentes de 93 pacientes, reclutando 31 pacientes/mes. En 8 casos no se obtuvo acceso a datos relevantes, excluyéndose, obteniendo muestra definitiva de 85 pacientes. De este total, 24 casos manifestaron RAM (28\%) y 61 pacientes no presentaron RAM (72\%). De los 24 pacientes que manifestaron RAM, hubo en total 48 medicamentos atribuibles a distintas manifestaciones de RAM: 58,3\% correspondió a antibióticos, 22,9\% a sedoanalgésicos, 10,4\% a antifúngicos y $8,3 \%$ a anticonvulsivantes (Figura 2). En cuanto a gravedad de la RAM, 72,9\% corresponden a gravedad moderada y $27,1 \%$ a gravedad intensa.

Los trastornos ocasionados por las RAMs, 


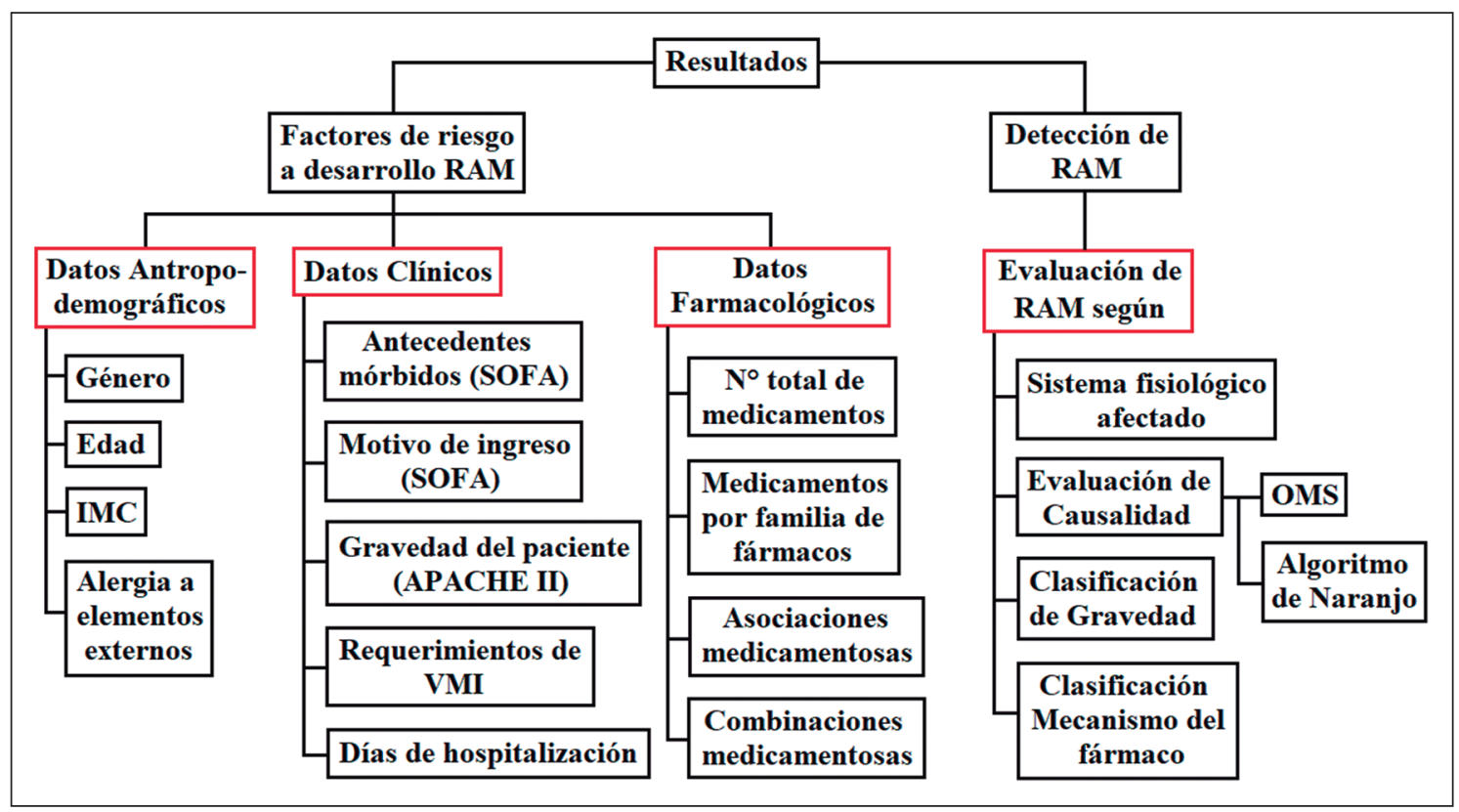

Figura 1. Organigrama conceptual de datos recopilados en el estudio. RAM: Reacción adversa a medicamentos. IMC: Índice de masa corporal. SOFA: Sequential Organ Failure Assessment. APACHE II: Acute Physiology and Chronic Health Evaluation II. VMI: ventilación mecánica invasiva. OMS: Organización Mundial de la Salud.

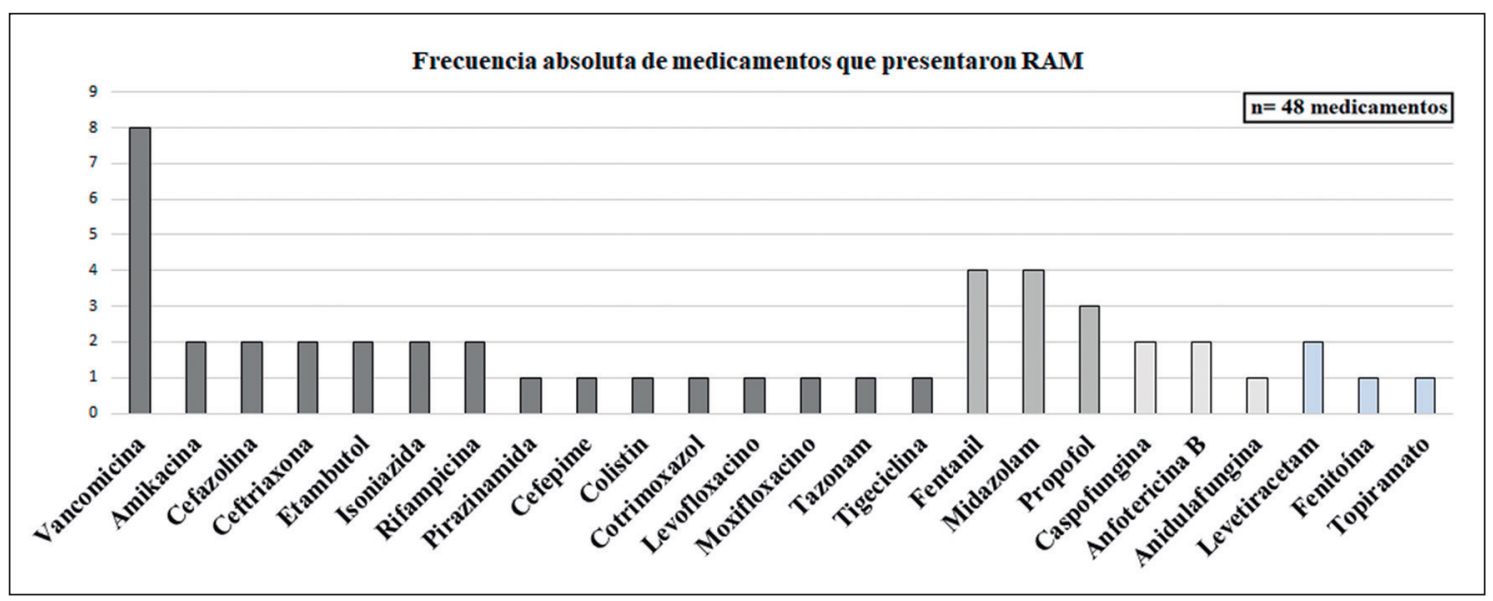

Figura 2. Frecuencia de medicamentos que presentaron RAM, ordenados por familia farmacológica.

20,8\% fueron trastornos de piel y anexos (eritema cutáneo, eritema máculo-papular, síndrome de cuello rojo con hiperemia ocular), trastornos hepáticos (incremento de transaminasas GOT/ GPT), trastornos neurológicos (delirum y crisis tónico clónico), y renales (incremento niveles de creatinina plasmática), cada uno con $14,6 \%$. Trastornos metabólicos (incremento de niveles de tri- glicéridos) en $8,3 \%$, alteraciones cardiovasculares (prolongación de intervalo QT), inmunológicas (síndrome febril e hipokalemia) y del sitio de aplicación (eritema cutáneo y petequias circunscritas al área de inyección), registraron 6,3\% para cada sistema. Finalmente, trastornos gastrointestinales (náuseas y emesis) y vasculares (trombocitopenia) en $4,1 \%$ para cada sistema (Figura 3 ). 


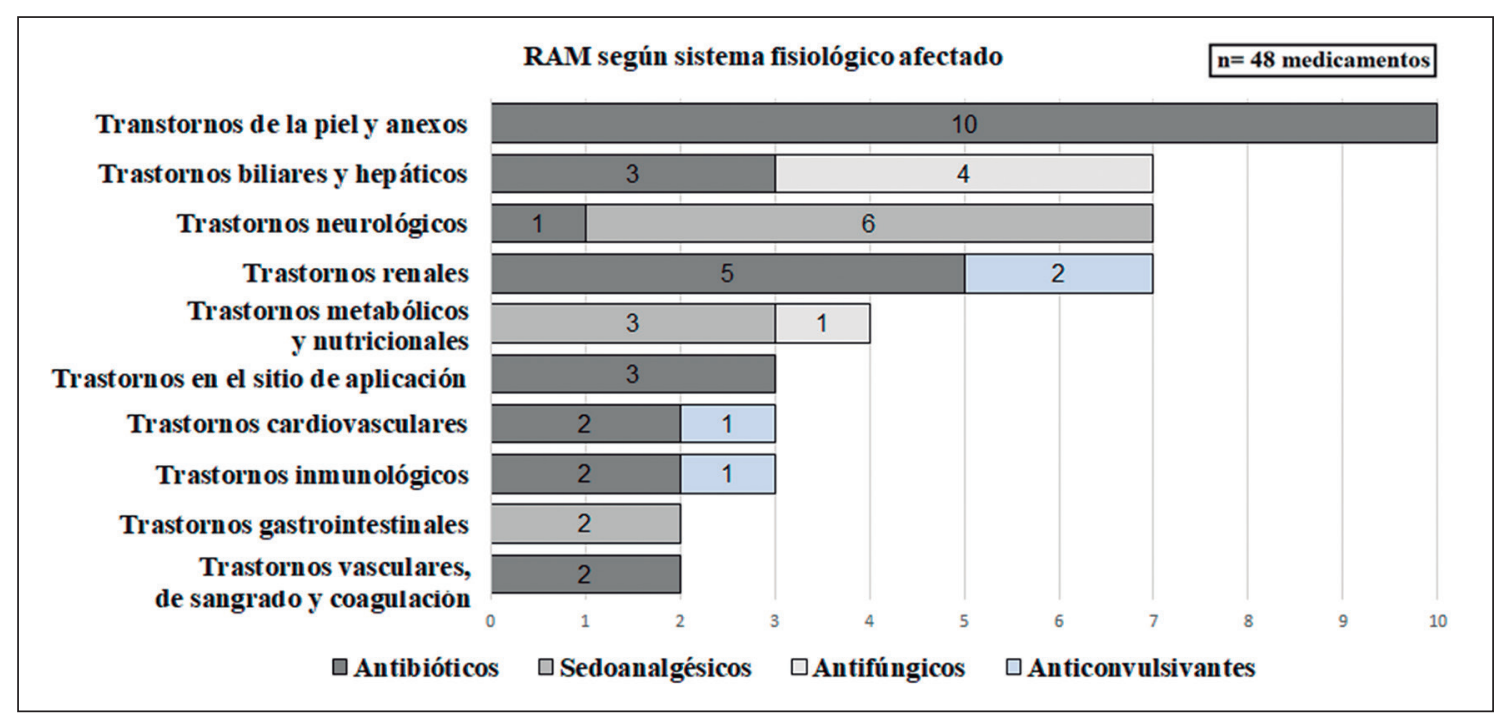

Figura 3. RAM reportadas según sistema fisiológico afectado por familia de medicamentos.

\section{Datos antropodemográficos}

Los pacientes que manifestaron RAM, 45,8\% correspondió a género femenino, edad promedio $54 \pm 37$ años, IMC promedio $28,5 \pm 11,3 \mathrm{Kg} /$ $\mathrm{m}^{2}$ y $29,2 \%$ refería alergias a medicamentos (betalactámicos, cefalosporinas, dipirona, medios de contrate) o a alimentos. Los pacientes que no desarrollaron RAM, 45,9\% eran mujeres, edad promedio $56 \pm 42$ años, IMC $24,9 \pm 8,2 \mathrm{Kg} / \mathrm{m}^{2}$ y solo el 6,5\% refirió antecedentes de alergias (medio de contraste, látex). No encontramos asociaciones estadísticamente significativas con variables género ni edad, sin embargo, frente a variables IMC en categoría sobrepeso-obesidad $\left[\chi^{2}(4)=16,19 \mathrm{p}<0,05\right.$ $(\mathrm{p}$-valor $=0,003)]$ y presencia de alergia $\left[\chi^{2}(1)=\right.$ $7,8, \mathrm{p}<0,05$ ( $\mathrm{p}$-valor $=0,005)]$, sí indican una asociación positiva con el desarrollo de RAM (Tabla 1).

\section{Datos clínicos}

Los antecedentes mórbidos y el motivo de ingreso a UCI se clasificaron según sistemas fisiológicos considerados en escala SOFA ${ }^{16}$, adicionando 2 categorías: sistema gastrointestinal y metabólico.

Los pacientes que desarrollaron RAM refirieron $3 \pm 2,4$ patologías como antecedentes mórbidos, los más afectados fueron sistema cardiovascular, metabólico y respiratorio. $\mathrm{Al}$ ingreso registraron $3 \pm 3,4$ sistemas fisiológicos comprometidos, los más relevantes: sistema respiratorio (sepsis de foco pulmonar, neumonía adquirida en la comunidad, neumonía asociada a ventilación mecánica, síndrome de distrés respiratorio agudo y otras), hematológico (coagulopatía por sepsis, principalmente), renal (insuficiencia renal aguda), cardiovascular (shock séptico mixto, shock cardiogénico, taquicardia ventricular) y hepático (descompensación de daño hepático previo, disfunción hepática por sepsis). No hubo ingresos por RAM. El APACHE II promedio fue $18 \pm 7,6$ puntos, el 79,2\% requirió VMI durante $6 \pm 4,4$ días y hospitalización de $11 \pm 8,4$ días, no registrándose prolongación de hospitalización por RAM.

De las 48 RAM pesquisadas, 36 se desarrollaron en pacientes sometidos a ventilación mecánica, $27,8 \%$ de las RAMs fueron de categoría grave, principalmente: trastornos cardiovasculares (prolongación de intervalo QT y arritmias), trastornos neurológicos (delirium y crisis tónico clónico), vasculares (trombocitopenia), metabólico (hipokalemia) y renales (incrementos de niveles de creatinina). El 72,2\% de las RAMs en pacientes con soporte ventilatorio, correspondió a categoría moderada: trastornos cutáneos, trastornos metabólicos (incrementos de triglicéridos $>200 \mathrm{mg} / \mathrm{dL}$ ) y trastornos inmunológicos (síndrome febril), entre otros.

Los pacientes que no desarrollaron RAM refirieron $2 \pm 2,9$ comorbilidades y $2 \pm 2,2$ sistemas fisiológicos comprometidos según motivo de ingreso, APACHE II de $10 \pm 10,5$ puntos, y solo el $22,9 \%$ requirió VMI por $3 \pm 4,4$ días, con hos- 
Tabla 1. Parámetros evaluados como factor de riesgo a desarrollo de RAM

\begin{tabular}{|c|c|c|c|c|c|}
\hline $\begin{array}{l}\text { Parámetros } \\
\text { evaluados }\end{array}$ & $\begin{array}{c}\text { Pacientes sin RAM } \\
(\bar{x} \pm D S)\end{array}$ & $\begin{array}{c}\text { Pacientes con RAM } \\
\text { ( } \bar{x} \pm \mathrm{DS})\end{array}$ & $\begin{array}{l}\text { T. Asociación } \\
\text { Valor } p^{(a)}\end{array}$ & $\begin{array}{c}\text { RR } \\
(\text { IC } 95 \%)^{(\mathbf{b})}\end{array}$ & $\begin{array}{l}\text { C. Spearman } \\
\text { rho }^{(c)}\end{array}$ \\
\hline Género & $\begin{array}{l}45,9 \% \text { Femenino } \\
54,1 \% \text { Masculino }\end{array}$ & $\begin{array}{l}\text { 45,8\% Femenino } \\
54,2 \% \text { Masculino }\end{array}$ & 0,788 & $\begin{array}{c}1 \\
(0,41-2,40)\end{array}$ & $-0,029$ \\
\hline Edad & $56 \pm 42$ años & $54 \pm 37$ años & 0,997 & $\begin{array}{c}1 \\
(0,25-1,87)\end{array}$ & $-0,055$ \\
\hline IMC & $24,9 \pm 8,2 \mathrm{Kg} / \mathrm{m}^{2}$ & $28,5 \pm 11,3 \mathrm{Kg} / \mathrm{m}^{2}$ & 0,003 & $\begin{array}{c}2,79 \\
(1,95-8,17)\end{array}$ & 0,337 \\
\hline $\begin{array}{l}\text { Antecedentes de } \\
\text { alergia }\end{array}$ & $\begin{array}{l}\text { 6,5\% Presencia } \\
93,5 \% \text { Ausencia }\end{array}$ & $\begin{array}{l}29,2 \% \text { Presencia } \\
70,8 \% \text { Ausencia }\end{array}$ & 0,005 & $\begin{array}{c}2,79 \\
(1,94-8,19)\end{array}$ & 0,303 \\
\hline $\begin{array}{l}\text { Antecedentes } \\
\text { mórbidos }\end{array}$ & $\begin{array}{c}2 \pm 2,98 \\
\text { patologías }\end{array}$ & $\begin{array}{c}3 \pm 2,44 \\
\text { patologías }\end{array}$ & 0,191 & $\begin{array}{c}1,32 \\
(0,67-2,71)\end{array}$ & $-0,181$ \\
\hline Motivo de ingreso & $\begin{array}{l}2 \pm 2,18 \\
\text { S. fisiológicos }\end{array}$ & $\begin{array}{l}3 \pm 3,36 \\
\text { S. fisiológicos }\end{array}$ & 0,002 & $\begin{array}{c}2,07 \\
(1,64-5,39)\end{array}$ & 0,293 \\
\hline $\begin{array}{l}\text { Gravedad paciente } \\
\text { (APACHE II) }\end{array}$ & $\begin{array}{l}10 \pm 10,5 \\
\text { puntos }\end{array}$ & $\begin{array}{l}18 \pm 7,6 \\
\text { puntos }\end{array}$ & 0,0001 & $\begin{array}{c}6,49 \\
(2,05-20,6)\end{array}$ & 0,583 \\
\hline $\begin{array}{l}\text { Requerimiento de } \\
\text { VMI }\end{array}$ & $\begin{array}{c}3 \pm 4,4 \text { días } \\
22,9 \% \text { VMI } \\
77,1 \% \text { No VMI }\end{array}$ & $\begin{array}{c}6 \pm 4,4 \text { días } \\
79,2 \% \text { VMI } \\
20,8 \% \text { No VMI }\end{array}$ & 0,001 & $\begin{array}{c}8,66 \\
(2,4-31,14)\end{array}$ & 0,651 \\
\hline $\begin{array}{l}\text { Días de } \\
\text { Hospitalización }\end{array}$ & $\begin{array}{l}4 \pm 5,8 \\
\text { días }\end{array}$ & $11 \pm 8,4$ días & 0,001 & $\begin{array}{c}2,47 \\
(2,68-24,1)\end{array}$ & 0,594 \\
\hline Medicamentos totales & $\begin{array}{c}11 \pm 8,02 \\
\text { medicamentos }\end{array}$ & $\begin{array}{c}21 \pm 9,8 \\
\text { medicamentos }\end{array}$ & 0,001 & - & 0,687 \\
\hline $\begin{array}{l}\text { Asociaciones } \\
\text { medicamentosas }\end{array}$ & $\begin{array}{c}2 \pm 2,4 \\
\text { medicamentos }\end{array}$ & $\begin{array}{c}5 \pm 3,8 \\
\text { medicamentos }\end{array}$ & 0,001 & - & 0,598 \\
\hline $\begin{array}{l}\text { Combinaciones } \\
\text { Medicamentosas }\end{array}$ & $\begin{array}{c}3 \pm 2,4 \\
\text { medicamentos }\end{array}$ & $\begin{array}{c}6 \pm 2,3 \\
\text { medicamentos }\end{array}$ & 0,001 & - & 0,512 \\
\hline
\end{tabular}

Donde: (a)T. Asociación: Test de asociación, $\chi^{2}$ : Chi- cuadrado, t: t de Student. Para $\chi^{2}$ y t asociación significativa $p<0,05$. (b)RR: Riesgo Relativo. RR: $<1$ riesgo negativo; $=1$ sin asociación; $>1$ riesgo positivo. ${ }^{(c)}$ C.S.: Correlación de Spearman. Para C. Spearman asociación significativa $p<0,05$; rho implica intensidad de asociación.

pitalizaciones de $4 \pm 5,8$ días. Todas las variables señaladas mostraron ser un parámetro de riesgo de desarrollo de RAM (Tabla 1), a excepción del parámetro antecedentes mórbidos $[t(83)=-1,32$; $\mathrm{p}>0,05(\mathrm{p}$-valor $=0,191)]$.

\section{Datos farmacológicos}

Se emplearon 1.133 fármacos durante la investigación, excluyéndose medicamentos empleados para tratamientos de RAMs. Se definió asociaciones medicamentosas a la administración concomitante de 2 o más medicamentos pertenecientes a la misma familia farmacológica ${ }^{20}$, mientras que, se comprende como combinaciones medicamentosas, a la administración de 2 o más fármacos correspondientes a distintas familias farmacológicas ${ }^{21}$.

Encontramos diferencias entre ambos subgru- pos de estudio, los pacientes que desarrollaron RAM recibieron administración de $21 \pm 9,8$ medicamentos, con media de $5 \pm 3,8$ asociaciones y $6+2,3$ combinaciones medicamentosas, mientras que pacientes que no desarrollaron RAM tuvieron tratamientos farmacoterapéuticos más acotados, con administración de $11 \pm 8,02$ medicamentos y una media de $2 \pm 2,4$ asociaciones y $3+2,36$ combinaciones medicamentosas.

Las asociaciones y combinaciones medicamentosas más frecuentes o graves, sospechosas de RAM se muestran en Tabla 2.

De las 48 RAMs pesquisadas, 28 correspondieron a antibióticos $(58,3 \%), 11$ a sedoanalgésicos (22,9\%), 5 a antimicóticos sistémicos $(10,4 \%)$ y 4 a anticonvulsivantes $(8,3 \%)$. Ver medicamentos en Figura 2. 
Tabla 2. Asociaciones y combinaciones medicamentosas con factor de riesgo a desarrollo de RAM

\begin{tabular}{|c|c|}
\hline Asociaciones medicamentosas & RAM Observada \\
\hline Cotrimoxazol* + Levofloxacino & Prolongación intervalo QT \\
\hline Isoniazida* + Rifampicina* + Etambutol* + Imipenem + Azitromicina & Toxicidad hepática \\
\hline Midazolam* + Fentanil & Delirium \\
\hline Propofol* + Fentanil+ Paracetamol & Incremento triglicéridos \\
\hline Topiramato* + Ácido Valproico & Síndrome febril \\
\hline Anfotericina B liposomal* + Voriconazol & $\begin{array}{l}\text { Hipokalemia } \\
\text { Incremento transaminasas }\end{array}$ \\
\hline Combinaciones medicamentosas & RAM Observada \\
\hline Cotrimoxazol* + Levofloxacino+ Enoxaparina + Paracetamol & Trombocitopenia \\
\hline Moxifloxacino* + Amiodarona+ Quetiapina & Prolongación intervalo QT \\
\hline
\end{tabular}

*Medicamento sospechoso de RAM.

Se señalan como factores de riesgo a desarrollar RAM: número total de medicamentos administra$\operatorname{dos}[t(83)=-9,46 ; \mathrm{p}<0,05(\mathrm{p}$-valor $=0,001)]$, asociaciones $[t(31)=-5,94 ; \mathrm{p} 0,05(\mathrm{p}$-valor $=0,001)]$ y combinaciones medicamentosas $[t(31)=-5,52$; $\mathrm{p}<0,05(\mathrm{p}$-valor $=0,001)]($ Tabla 1$)$.

\section{Intensidad de riesgo a desarrollo de RAM}

Los parámetros con mayor intensidad como factor de riesgo para desarrollo de RAM, descritas en orden decreciente, fueron: número de medicamentos administrados: $r h o=0,687 ; \mathrm{p}<0,05$ $(\mathrm{p}$-valor $=0,001) ; \mathrm{VMI}$ : rho $=0,651 ; \mathrm{p}<0,05$ ( $\mathrm{p}$-valor $=0,001)$; asociaciones medicamentosas: rho $=0,598 ; \mathrm{p}<0,05(\mathrm{p}$-valor $=0,001)$; hospitalización $>$ a 7 días: $r$ ho $=0,594 ; \mathrm{p}<0,05$ ( $\mathrm{p}$-valor $=0,001)$; APACHE II $\geq$ a 14 puntos: rho $=0,583 ; \mathrm{p}<0,05(\mathrm{p}$-valor $=0,0001)$; combinaciones medicamentosas: rho $=0,512$; $\mathrm{p}<0,05$ ( $\mathrm{p}$-valor $=0,001) ;$ IMC en categoría sobrepeso-obesidad: rho $=0,337 ; \mathrm{p}<0,05(\mathrm{p}-$ valor $=0,02)$; antecedentes de alergia: $r h o=0,303$; $\mathrm{p}<0,05(\mathrm{p}$-valor $=0,005)$ y motivo de ingreso $(\mathrm{SOFA})$ : $[\mathrm{rho}=0,293 ; \mathrm{p}<0,05(\mathrm{p}$-valor $=0,006)]$.

\section{Evaluación de causalidad y clasificación RAM}

Ambos métodos de evaluación de causalidad presentaron una correlación moderada, con buen nivel de concordancia [rho $=0,637 ; \mathrm{p}<0,05$ ( $\mathrm{p}$ - valor $=0,0001)]$, mas no suficiente para ser reemplazables entre sí, puesto que $\alpha$ de Cronbach manifiesta una fiabilidad moderada.

Los métodos de clasificación de RAM según gravedad y mecanismo del fármaco mostraron escasa concordancia $[\mathrm{rho}=0,498 ; \mathrm{p}<0,05(\mathrm{p}$ valor $=0,04)]$, corroborado por coeficiente $\alpha$ de Cronbach $(0,632)$, implicando que ambos métodos señalan una fiabilidad cuestionable entre sí (Tabla 3).

\section{Discusión}

Del total de pacientes, $28 \%$ manifestó al menos una RAM, denotando elevada incidencia, siendo relevante contar con un profesional encargado de farmacovigilancia, que oriente seguras prescripciones y reportes, además, optimice el servicio de atención hospitalaria ${ }^{6}$.

Respecto a factores de riesgo antropodemográficos, se observó que las variables IMC en categoría sobrepeso-obesidad [2,79 (IC 95\% 1,95-8,17)] y presencia de alergia 2,77 (IC 95\% 1,94-8,19) indican una asociación estadísticamente significativa, recalcando la relevancia de parámetros farmacocinéticos ${ }^{23}$ y reacción de hipersensibili$\mathrm{dad}^{22}$ en el desarrollo de RAM. Los pacientes con IMC elevado poseen aumento de tejido graso y disminución de agua corporal total, generando cambios en el volumen de distribución normal de medicamentos, además, el aumento de ácidos grasos libres obstaculiza la unión a proteínas plasmáticas (UPP), generando una mayor concentración plasmática de fármaco libre ${ }^{23,24}$. Por otra parte, la infiltración de grasa hepática, disminuye la metabolización de fármacos, resultando en menor biotransformación de estos ${ }^{23}$.

En cuanto a variables clínicas, motivo de ingre- 
Tabla 3. Análisis de métodos de evaluación de causalidad y clasificación de RAM reportadas

\begin{tabular}{|c|c|c|c|c|c|}
\hline Evaluación/Clasificación & Categoría & $\%$ & Valor P rho(d) & Kruskall Wallis K(e) & $\alpha$ Cronbach $^{(f)}$ \\
\hline \multirow[t]{3}{*}{ Causalidad de la OMS } & Cierta & 35,4 & 0,0001 & 0,604 & 0,779 \\
\hline & Probable & 58,3 & 0,637 & & \\
\hline & Posible & 6,3 & & & \\
\hline \multirow[t]{3}{*}{ Causalidad A. de Naranjo } & Definitiva & 31,3 & & & \\
\hline & Probable & 54,2 & & & \\
\hline & Posible & 14,5 & & & \\
\hline \multirow[t]{2}{*}{ Clasificación gravedad } & Moderada & 72,9 & 0,04 & 0,389 & 0,632 \\
\hline & Grave & 27,1 & 0,498 & & \\
\hline \multirow{2}{*}{$\begin{array}{l}\text { Clasificación Mecanismo del } \\
\text { fármaco }\end{array}$} & $A$ & 75 & & & \\
\hline & $B$ & 25 & & & \\
\hline
\end{tabular}

Donde: (d) Correlación de Spearman. Para C. Spearman asociación significativa $\mathrm{p}<0,05$; rho implica intensidad de asociación. Correlación positiva moderada; 0,40-0,69. (e)K: nivel de concordancia. Ínfima: 0-0,2; Escasa: 0,21-0,4; Moderada: 0,410,6; Buena: 0,61-0,8; Muy Buena: 0,81-1. ${ }^{(\mathrm{f})} \alpha$ : grado confiabilidad. Inaceptable $<0,5$; Pobre $>0,5$; Cuestionable $>0,6$; Aceptable $>0,7$; Buena $>0,8$; Excelente $>0,9$.

so (SOFA) $[2,07$ (IC 95\% 1,64-5,39)] y APACHE II $\geq 14$ puntos $[6,49$ (IC 95\% 2,05- 20,62)] interfieren en que patologías con marcado impacto a sistemas fisiológicos relevantes en la participación de parámetros farmacocinéticos son factores de riesgo a desarrollar $\mathrm{RAM}^{25,29}$. El requerimiento de VMI limita biotransformaciones de fase I dependientes de oxígeno, por incremento de presión intratorácica, con el consecuente aumento de presión intraabdominal y reducción del flujo portal $^{28}$. Asimismo, extensas hospitalizaciones generan cambios fisiológicos, intensificando la probabilidad de RAM ${ }^{24,29}$.

Respecto a datos farmacológicos, todos los pacientes se encontraban en categoría de polifarmacia. La administración de 10 a 20 medicamentos aumenta la probabilidad de interacción en un $20 \%$, predisponiendo a $\mathrm{RAM}^{30}$. La gran diferencia en el número de medicamentos administrados a pacientes que desarrollaron RAM era esperada, dado el elevado APACHE II y prolongada permanencia en UCI ( $\overline{\mathrm{x}}=11$ días $)$.

Las asociaciones más relevantes en el desarrollo RAM en nuestra serie destacan: Cotrimoxazol-levofloxacino prolongando intervalo QT, notificándose interacciones entre quinolonas y sulfonamidas originando arritmias ventriculares ${ }^{31,35}$. La asociación isoniazida-rifampicina-etambutol-imipenem-azitromicina, generó toxicidad hepática. Rifampicina aumenta la toxicidad de isoniazida por incremento de metabolismo, produciéndose más metabolitos hepatotóxicos, conllevando a RAM observada ${ }^{31,35}$.

La asociación de sedoanalgésicos midazolam-fentanil, originó en 4 oportunidades delirium. Con esta asociación, se debe tener precaución, en particular, con el uso prolongado en infusión ${ }^{31,32}$.

La asociación propofol-fentanil-paracetamol presenta incremento en niveles de triglicéridos, fundamentado en la composición intrínseca de propofol, el que es potenciado por competencia de UPP entre fentanil-propofol, ocasionado mayor cantidad de Propofol libre en plasma. Además, entre paracetamol-propofol se prioriza la biotransformación de paracetamol, elevando concentraciones de propofol ${ }^{32-35}$.

Otra asociación de relevancia clínica correspondió a topiramato-ácido valproico, implicando síndrome febril. La administración conjunta de ácido valproico y topiramato incrementa la toxicidad del otro fármaco, se sugiere administración aislada de ambos fármacos ${ }^{31,35}$. Por último, la asociación anfotericina B liposomal-voriconazol, generó hipokalemia e incremento de niveles de transaminasas, no reporta interacciones existentes $^{35}$, no obstante, se sospecha de un incremento de los niveles de anfotericina B liposomal, dado el mecanismo de voriconazol, que concomitantemente inhibe citocromo P450, aumentando concentraciones séricas de anfotericina, resultando, en ocasiones, incremento de permeabilidad y destrucción de células tubulares renales, conllevando 
a pérdida renal de potasio, efecto reversible al suspender la administración ${ }^{35,36}$. Además, la elevada afinidad por ergosterol-colesterol genera injuria celular de hepatocitos, resultando en incremento de transaminasas ${ }^{31}$.

Una combinación relevante fue amiodarona-quetiapina-moxifloxacino, prolongando intervalo QT (QT/QTcb: 393/487 ms) posterior a la administración de moxifloxacino. Se ha reportado interacción entre amiodarona-moxifloxacino y quetiapina-moxifloxacino, generando prolongación de intervalo QT, arritmias ventriculares potencialmente graves, de modo que, se sugiere evitar administración concomitante entre estos fármacos ${ }^{31,35}$.

Finalmente, respecto a evaluación según causalidad de $\mathrm{OMS}^{14} \mathrm{y}$ algoritmo de Naranjo ${ }^{15}$, no son evaluaciones reemplazables entre sí, puesto que la causalidad según $\mathrm{OMS}^{14}$ se basa en definiciones clínicas, extensas y poco precisas, implicando errores de interpretación, mientras, la evaluación según algoritmo de Naranjo ${ }^{15}$ parece ser más precisa, pues está fundamentada en preguntas con puntajes, siendo una evaluación de carácter más objetivo. Por último, las clasificaciones de RAM focalizada en gravedad ${ }^{10}$ y mecanismo de acción del fármaco ${ }^{19}$ tienen una correlación moderada $[$ rho $=0,498 ; \mathrm{p}<0,05(\mathrm{p}$-valor $=0,04)]$, mas no señala un nivel de concordancia bueno $[\mathrm{k}=0,389$; $\mathrm{p}<0,05(\mathrm{p}$-valor $=0,041)]$, pues son métodos con distinta orientación, uno centrado en la gravedad ocasionada al paciente y otro en mecanismo del fármaco.

Debemos destacar que, para una certera relación de causalidad se requiere de la reexposición del fármaco sospechoso y constatar la aparición de la RAM $^{14}$, no obstante, dado el delicado estado de salud y, con el objetivo de no ocasionar un deterioro en la evolución clínica del paciente, no se readministró el medicamento sospechoso, considerando estrictamente las definiciones establecidas por métodos de causalidad por parte de los evaluadores.

\section{Conclusión}

Las RAMs corresponden a un evento clínico prevenible, que ocurre con una incidencia significativa en UCI y las variables de riesgo presentan gran impacto en parámetros farmacocinéticos y farmacodinámicos, siendo relevantes para la prevención de estas. Los sistemas de causalidad y clasificación de RAM presentan ciertas concordancias entre sí, mas no son reemplazables, comprendiendo que son métodos con distintos focos de evaluación.

\section{Referencias}

1. Cortés A, Sáez E. Criterios de admisión y egreso para unidades de pacientes críticos de adultos. En: Ugarte S, Tomicic V, Marks R, editores. Emergencias Médicas Y Paciente Crítico. $2^{\mathrm{a}}$ ed. Santiago, Chile: Editorial Mediterráneo Ltda.; 2017. p. 616-32.

2. Cornejo R. Estructura, función y gestión en cuidados intensivos. En: Andresen M, editor. Manual de Medicina Intensiva. $2^{\mathrm{a}}$ ed. Santiago, Chile: Editorial Mediterráneo Ltda.; 2013. p. 15-26.

3. Comité de Consenso. Tercer consenso de Granada sobre problemas relacionados con medicamentos (PRM) y resultados negativos asociados a la medicación (RNM). Ars Pharm 2007; 48 (1): 5-17.

4. Gastelurrutia MA, Faus MJ, Martínez-Martínez F. Resultados negativos asociados a la medicación. Ars Pharm 2016; 57 (2): 89-92.

5. Pérez Menéndez-Conde C, Bermejo Vicedo T, Delgado Silveira E, Carretero Accame E. Resultados negativos asociados al uso de medicamentos que motivan ingreso hospitalario. Farm Hosp 2011; 35 (5): 236-43.

6. OMS. La farmacovigilancia: garantía de seguridad en el uso de los medicamentos. Perspect políticas la OMS sobre Medicam 2004; 9: 1-6.

7. Sánchez I, Amador C, Plaza JC, Correa G, Amador R. Impacto clínico de un sistema de farmacovigilancia activa realizado por un farmacéutico en el reporte y subnotificación de reacciones adversas a medicamentos. Rev Med Chile 2014; 142 (8): 998-1005.

8. Lazarou J, Pomeranz BH, Corey PN. Incidence of adverse drug reactions in hospitalized patients: a meta-analysis of prospective studies. JAMA 1998; 279 (15): 1200-5.

9. Bond CA, Raehl CL. Adverse drug reactions in united states hospitals. Pharmacotherapy 2006; 26 (5): 601-8.

10. Telechea H, Speranza N, Lucas L, Giachetto G, Nanni L, Menchaca A. Reacciones adversas a medicamentos en una unidad de cuidados intensivos pediátrica. Farm Hosp 2012; 36 (5): 403-9.

11. González C, Astargo C, Curilén N, Escobar L, Alvarado $\mathrm{S}$. Caracterización de las reacciones adversas a medicamentos registrados por el programa de farmacovigi- 
lancia de un hospital pediátrico. 2011-2014. Rev Chile Pediatr 2015; 86(2): 84-89.

12. Rangel CR, Rojas S, López M, Caviedes G. Prevalencia y factores asociados a la presencia de reacciones adversas a medicamentos en los pacientes tratados por medicina interna en el Hospital Universitario Hernando Moncaleano Perdomo. Rev Méd. Risaralda 2016; 22 (2): 87-90.

13. Pérez R, Gil L. Selección de los 12 medicamentos más utilizados en los servicios de urgencia y cuidados intensivos. Rev Cubana Med Milit 2001; 30 (5): 97-103.

14. Kyonen M, Folatre I, Lagos X, Vargas S. Comparación de dos métodos de evaluación de causalidad de sospechas de reacciones adversas a medicamentos (RAM). 2003-2009. Rev Med Chile 2015; 143 (7): 880-6.

15. Naranjo CA, Busto U, Sellers EM, Sandor P, Ruiz I, Roberts EA, et al. A method for estimating the probability of adverse drug reactions. Clin Pharmacol Ther 1981; 30 (2): 239-45.

16. Jones AE, Trzeciak S, Kline JA. The Srquential Organ Failure Assessment score for predicting outcome in patients with severe sepsis and evidence of hyperperfusion at the time of emergency department presentation. Crit Care Med. 2009; 37(5): 1649-54.

17. Olsson T, Lind L. Comparison of the rapid emergency medicine score and APACHEII in nonsurgeical emergency department patients. Acad Emerg Med. 2003; 10(10): 1040-8.

18. Salas S, Pérez M, Meléndez S. Farmacovigilancia intensiva en el servicio de medicina interna del Hospital Regional No.1 del Instituto Mexicano del Seguro Social en Tijuana. Rev Mex de Ciencias Farmacéuticas 2012; 43 (4): 55-68.

19. Orellana R. Sistema de farmacovigilancia en el Hospital Clínico Universidad de Chile. Medwave 2011; 11(8): e5111. Disponible en: www.medwave.cl/link.cgi/Medwave/Revisiones/RevisionTemas/5111 [Consultado el 13 de abril de 2021]

20. Plaza J, Álamo M, Torres P, Fuentes A, López F. Interacciones de medicamentos y eventos adversos en fármacos utilizados en una unidad de cuidados intensivos. Rev Med Chile 2010; 138 (3): 452-60.

21. Tarigo J, Viroga S, Amigo C, López M, Speranza N. Algunos ejemplos de medicamentos de amplio uso y escaso valor terapéutico I: combinaciones a dosis fija. Boletín Farmacología Uruguay 2015; 6 (1): 16-23.

22. Schrijvers R, Gilissen L, Chiriac AM, Demoly P. Pathogenesis and diagnosis of delayed- type drug hypersensitivity reactions, from bedside to bench and back. Clin Transl Allergy 2015; 5 (31): 1-10.

23. Cheymol G. Effects of obesity on pharmacokinetics im- plications for drug therapy. Clin Pharmacokinet 2000; 39 (3): 215-31.

24. Brill MJ, Diepstraten J, van Rongen A, van Kralingen $S$, van den Anker JN, Knibbe CA. Impact of obesity on drug metabolism and elimination in adults and children. Clin Pharmacokinet 2012; 51 (5): 1-10.

25. Villagra S. Seguimiento farmacoteraéutico y farmacocinético en UCI. En: Ugarte S, Tomicic V, Marks R, editores. Emergencias Médicas y Paciente Crítico. $2^{\mathrm{a}}$ ed. Santiago, Chile: Editorial Mediterráneo Ltda.; 2017. p. 672-81.

26. Lagneau F, Perbet S, Delefosse D, Wernet A, Stocco J, Marty J. Drugs pharmacokinetics in ICU patients: consequences of hypoalbuminemia upon drugs monitoring and dosing scheme. Intensive Care Med 2004; 30 (6): 1247.

27. Power BM, Forbes AM, van Heerden P, Ilett KF. Pharmacokinetics of drugs used in critically ill adults. Clin Pharmacokinet 1998; 34 (1): 25-56.

28. García Castillo E, Chicot Llano M, Rodríguez Serrano DA, Zamora García E. Ventilación mecánica no invasiva e invasiva. Med - Programa Form Médica Contin Acreditado 2014; 11 (63): 3759-67.

29. Regueira T. Consideraciones farmacológicas generales y particulares en cuidados intensivos. Rev Médica Clínica Las Condes 2016; 27 (5): 636-45.

30. Depont F, Vargas F, Dutronc H, Giauque E, Ragnaud J-M, Galpérine T, et al. Drug-drug interactions with systemic antifungals in clinical practice. Pharmacoepidemiol Drug Saf 2007; 16 (11): 1227-33.

31. Medscape. Drug interaction checker tool [Internet]. WebMD LLC. Disponible en: reference.medscape.com/ drug-interactionchecker [Consultado el 23 de octubre de 2020]

32. Balas MC, Weinhouse GL, Denehy L, Chanques G, Rochwerg B, Misak CJ, et al. Interpreting and Implementing the 2018 pain, agitation/sedation, delirium, immobility, and sleep disruption clinical practice guideline. Crit Care Med 2018; 46 (9): 1464-70.

33. Rosas-Gutiérrez I, Simón-Arceo K, Mercado F. Mecanismo celular y molecular de la adicción a benzodiacepinas. Salud Ment 2013; 36 (4): 325-9.

34. Galeotti G. Farmacocinética del propofol en infusión. Educ Contin 2009; 67 (2): 154-85.

35. Micromedex. Web Aplications [Internet]. IBM Corporation. 2020. Disponible en: www.micromedexsolutions. com/home/dispatch [Consultado el 20 de octubre de 2020].

36. Patel R. Antifungal agents. Part 1. Amphotericin B preparations and flucytocine. Mayo Clinic Proceedings. 1998; 73 (12):1205-25. 